\title{
MOTION OF A CHARGED PARTICLE IN A FIELD OF PLANE-WAVE ELECTROMAGNETIC RADIATION*
}

\author{
By J. L. SYNGE (Dublin Institute for Advanced Studies)
}

\begin{abstract}
A charged particle is acted on by plane waves of electromagnetic radiation, in general not monochromatic. In the absence of radiation damping, the relativistic equations of motion are solved exactly by quadratures. Radiation damping is then treated as perturbation.
\end{abstract}

1. Plane waves of electromagnetic radiation. Let Latin indices range $1,2,3,4$. Let the speed of light be unity. Let the coordinates be $x_{r}$ with $x_{4}=i t$, so that the element $d \tau$ of proper time on the world line of a particle is given by $d \tau^{2}=-d x_{r} d x_{r}$.

A Maxwellian field in vacuo satisfies the eight equations

$$
F_{r, \text { e }}=0, \quad F_{r, t}+F_{\text {st }, r}+F_{t r, \text { }}=0,
$$

where the comma indicates partial differentiation. We are interested only in fields which correspond to plane waves of radiation. To describe these, it is convenient to introduce the variables

$$
\xi=x_{1}-t=x_{1}+i x_{4}, \quad \eta=x_{1}+t=x_{1}-i x_{4} .
$$

The first criterion for plane waves propagated in the direction of the $x_{1}$-axis is that all the components $F_{r s}$ are functions only of $\xi$. Then the eight equations (1.1) reduce to four:

$$
F_{14}^{\prime}=0, \quad F_{23}^{\prime}=0, \quad F_{21}^{\prime}+i F_{24}^{\prime}=0, \quad F_{31}^{\prime}+i F_{34}^{\prime}=0,
$$

where the prime means $d / d \xi$. On integrating we get four constants of integration. The second criterion for plane waves is that these constants should vanish, so that we have

$$
F_{14}=0, \quad F_{23}=0, \quad F_{21}+i F_{24}=0, \quad F_{31}+i F_{34}=0 .
$$

To convert these to the more usual $(E, H)$ notation, we are to put

$$
E_{1}=i F_{14}, \quad H_{1}=F_{23},
$$

with cyclic permutation of $(1,2,3)$. Thus we get

$$
E_{1}=0, \quad H_{1}=0, \quad H_{2}=-E_{3}, \quad H_{3}=E_{2},
$$

and so recognise the usual picture of radiation, with the two vectors lying in the plane of the wave, perpendicular to each other and of equal magnitudes. But note that there is no suggestion of sinusoidal character. It is true that any such wave may be resolved into an infinity of sinusoidal waves, but that is not useful in the prosent problem in view of thic nonlinear nature of the equations of motion of a charged particle.

\footnotetext{
* Received December 22, 1969.
} 
The essential point about the radiation field (1.6) is that it is defined by two functions $E_{2}(\xi), E_{3}(\xi)$ which are quite arbitrary except for such conditions of smoothness as may be required in the subsequent argument.

2. Equations of motion in general. For a ch:trged particle (mass $m$, charge $e$ ) moving in a given external electromagnetic field $F_{r s}$, the equations of motion are

$$
m \dot{v}_{r}=e F_{r s} v_{s}+K_{r} .
$$

The dot means $d / d \tau$ and $v_{r}$ is the 4-velocity $\left(d x_{r} / d \tau\right)$. The final term is

$$
K_{r}=k\left(\ddot{v}_{r}-v_{r} \dot{v}_{n} \dot{i}_{n}\right) \text {; }
$$

if we put $k=e^{2} / 6 \pi$, we have the usual damping term, and if we put $k=0$ we omit the damping. Since in the following argument we have no occasion to divide by $k$, it is a matter of indifference whether it vanishes or not. In other words, we proceed as if damping were present, but we can drop the damping whenever we like.

The world line of the particle, being timelike, is bound to meet the hyperplane $\xi=0$. There is no loss of generality in taking that event for the origin of coordinates and in measuring the proper time from that same event. Thus, we have as an initial condition

$$
x_{r}=0 \text { for } \tau=0 .
$$

This initial condition is, of course, not enough to determine the motion. If $k=0$, we need also the initial $v_{r}$; if $k \neq 0$, we need that and also the initial $\dot{v}_{r}$.

We are now to use the radiation conditions (1.4) in the equations of motion. When those conditions are translated as in (1.5), (2.1) become

$$
\begin{array}{ll}
m \dot{v}_{1}=e\left(E_{2} v_{2}+E_{3} v_{3}\right)+K_{1}, & m \dot{v}_{2}=-e E_{2}\left(v_{1}+i v_{4}\right)+K_{2}, \\
m \dot{v}_{3}=-e E_{3}\left(v_{1}+i v_{4}\right)+K_{3}, & m \dot{v}_{4}=i e\left(E_{2} v_{2}+E_{3} v_{3}\right)+K_{4} .
\end{array}
$$

By (1.2) we have

$$
v_{1}+i v_{4}=\dot{\xi}, \quad v_{1}-i v_{4}=\dot{\eta},
$$

and so, combining the first and last of (2.4), we get the four equations

$$
\begin{aligned}
m \ddot{\xi}=K_{1}+i K_{4}, \quad m \ddot{\eta}= & 2 e\left(E_{2} v_{2}+E_{3} v_{3}\right)+K_{1}-i K_{4}, \\
& m \dot{v}_{2}=-e E_{2} \dot{\xi}+K_{2}, \quad m \dot{v}_{3}=-e E_{3} \dot{\xi}+K_{3} .
\end{aligned}
$$

3. Motion without damping. Iet us now drop the damping term by putting $k=0$. Then the $K$-terms disappear from (2.6). In view of the initial condition (2.3), the first of (2.6) gives

$$
\dot{\xi}=-a, \quad \xi=-a \tau,
$$

where $a$ is a constant. If $\mathbf{u}$ is the 3 -velocity of the particle $\left(u_{1}=d x_{1} / d t\right.$, etc.), the first of (2.5) tells us that

$$
a=\gamma\left(1-u_{1}\right), \quad \gamma=\left(1-u^{2}\right)^{-1 / 2} .
$$

This is an integral of the motion; note that $a$ is necessarily positive since $u^{2}<1$.

The formula (3.1) is the key to the integration process. The main difficulty in solving 
the equations of motion lies in the fact that the elements of the motion are functions of $\tau$, whereas the field is a function of $\xi$. Now we see that these two variables are simply related as in (3.1).

The third of (2.6) now reads

$$
m \dot{v}_{2}=a e E_{2}(\xi)=a e E_{2}(-a \tau),
$$

and the fourth is similar. Thus

$v_{2}(\tau)=a(e / m) \int_{0}^{\tau} E_{2}(-a p) d p+b_{2}, \quad v_{3}(\tau)=a(e / m) \int_{0}^{\tau} E_{3}(-a p) d p+b_{3}$,

where $b_{2}$ and $b_{3}$ are constants, viz. the initial values of $v_{2}$ and $v_{3}$.

The functions $v_{2}(\tau)$ and $v_{3}(\tau)$ being now known, we turn to the second of (2.6) and get

$$
\dot{\eta}=2(e / m) \int_{0}^{\tau}\left[E_{2}(-a p) v_{2}(p)+E_{3}(-a p) v_{3}(p)\right] d p+c,
$$

where $c$ is a constant of integration.

In (3.1), (3.4), and (3.5), we now have essentially the four components of the 4velocity of the particle. There are four constants of integration, $a, b_{2}, b_{3}, c$, but they are not independent. We have the identity

$$
v_{r} v_{r}=-1
$$

or equivalently

$$
v_{2}^{2}+v_{3}^{2}+\dot{\xi} \dot{\eta}=-1 .
$$

When we substitute from the above equations for $\tau=0$, we get

$$
b_{2}^{2}+b_{3}^{2}-a c=-1 \text {. }
$$

Having found the 4-velocity as above, it needs only a further integration to obtain the trajectory $x_{r}=x_{r}(\tau)$.

4. The damping term as a perturbation. I Let us go back to the exact equations (2.6) and treat the damping terms as a perturbation. The plan will be to treat the factor $k$ in (2.2) as small. Then (2.6) gives

$$
\dot{v}_{n} \dot{v}_{n}=\dot{v}_{2}^{2}+\dot{v}_{3}^{2}+\ddot{\xi} \ddot{\eta}=(e E / m)^{2} \dot{\xi}^{2}+O(k),
$$

where $E$ is the magnitude of the electric vector, i.e.

$$
E(\xi)=\left(E_{2}^{2}+E_{3}^{2}\right)^{1 / 2} .
$$

It is to be understood that the error term $O(k)$ in (4.1), and other such terms below, may grow with time, and the perturbation method is valid only for a finite range of $\tau$, or, in general terms, for a range of $\tau$ such that $k \tau$ is small.

It is convenient to define the (positive) function of $\xi$,

$$
Q(\xi)=[a e E(\xi) / m]^{2} .
$$

Then, since according to $(3.1)_{\lambda} \dot{\xi}=-a$ in the unperturbed motion, (4.1) gives

$$
\dot{v}_{n} \dot{v}_{n}=Q(\xi)+O(k)
$$


By (2.2) we have

$$
K_{1}+i K_{4}=k(\dddot{\xi}-\xi Q)+O\left(k^{2}\right)=k a Q(\xi)+O\left(k^{2}\right),
$$

and so the first of (2.6) reads

$$
m \ddot{\xi}=k a Q(-a \tau)+O\left(k^{2}\right) .
$$

Therefore

$$
\dot{\xi}=-a+(k a / m) \int_{0}^{r} Q(-a p) d p+O\left(k^{2}\right),
$$

in which the integral is positive for positive $\tau$, but of course small compared with the leading term. A further integration gives $\xi$.

As for the other three equations in (2.6), we have

$$
\begin{aligned}
K_{1}-i K_{4} & =k(\dddot{\eta}-\dot{\eta} Q)+O\left(k^{2}\right), \\
K_{2} & =k\left(\ddot{v}_{2}-v_{2} Q\right)+O\left(k^{2}\right), \quad K_{3}=k\left(\ddot{v}_{3}-v_{3} Q\right)+O\left(k^{2}\right),
\end{aligned}
$$

in which the unperturbed values are to be inserted. The third of (2.6) reads

$$
m \dot{v}_{2}=-e E_{2}(\xi) \dot{\xi}+K_{2} ;
$$

here we are to substitute for $\xi$ and $\xi$ the values given by (4.7) and for $K_{2}$ the value from (4.8); the right-hand side is then a known function of $\tau$, and one integration gives $v_{2}$; a second integration gives $x_{2}$. Similarly we get $v_{3}$ and $x_{3}$. As for $\eta$, we can now get it from (2.6) by two integrations, the right-hand side being known.

In all these results there is an error $O\left(k^{2}\right)$. To within such an error, the effect of the small damping term has been found.

5. Conclusion. Vachaspati [1] showed that the motion of a charged particle may be found accurately when the radiation field is monochromatic and the damping term is omitted. The present paper may be regarded as a generalisation of his work.

\section{REFERENCE}

[1] Vachaspati, Exact solution of relativistic equations of motion of an electron in an external radiation field, Proc. Nat. Inst. Sci. India Part A 29, 138-142 (1963) 